\title{
Micro e pequenas empresas no turismo: aspectos teórico-conceituais
}

\section{Micro and small business in Tourism: theoretical and conceptual aspects}

\author{
Kleber de Oliveira da Silva (SILVA, K. de O. da) ${ }^{*}$ e \\ Sara Joana Gadotti dos Anjos (ANJOS, S. J. G. dos) ${ }^{* *}$
}

RESUMO - O presente trabalho tem como objetivo principal analisar a produção científica sobre micro e pequenas empresas (MPE) no turismo no contexto brasileiro e internacional entre 1990 a 2014. Para a identificação dos artigos, foram consultadas duas bases de dados (ScienceDirect e EBSCO) e duas editoras (SAGE e Emerald), que indexam e publicam os principais periódicos de turismo na língua inglesa no mundo. No caso dos periódicos Brasileiros, utilizou-se como referência o website "Publicações de Turismo", que indexa as principais revistas científicas brasileiras de turismo. O levantamento resultou em 196 artigos consultados (21 brasileiros e 175 internacionais). Para cada artigo foram utilizados parâmetros de análise quantitativos e qualitativos: a evolução das pesquisas na área; as principais temáticas pesquisadas; definições de MPE adotadas; empresas objetos de estudo e localidades estudadas. Os resultados apontaram a necessidade do desenvolvimento de uma base teórica mais densa sobre o tema, além de uma análise de MPE no turismo de um modo mais analítico, ressaltando as características da realidade local, na qual essas empresas estão inseridas. Também é apresentado um panorama das principais contribuições dos estudos sobre micro e pequenas empresas no Brasil e no exterior.

Palavras-chave: Turismo; Micro e pequenas empresas; Análise bibliométrica; Estado da arte.

ABSTRACT - This study aims to review scientific literature on micro and small business (MSBs) in Tourism in Brazilian and international context from 1990 to 2014. In order to identify articles were consulted two databases (EBSCO and ScienceDirect) and two publishers (SAGE and Emerald), that index and publish the main tourism journals in English. In the case of Brazilian journals, it was used the website "Publicações de Turismo" as reference, since it index the Brazilian Tourism major journals. The survey resulted in 196 articles consulted (21 Brazilian and 175 international). For each article were used quantitative and qualitative analysis parameters: the evolution of research in the area; the main topics researched; MSBs definitions adopted; tourism sectors and locations studied. The results indicated the need to develop a more consistent theoretical basis on the subject, and a MSB analysis

\footnotetext{
* Formação: Graduação em Turismo (Bacharelado) pela Escola de Comunicação e Artes da Universidade de São Paulo (ECA-USP) e Mestrado em Turismo e Hotelaria pela Universidade do Vale do Itajaí (UNIVALI). Endereço físico para correspondência: Rua Rio Paraná, 403. CEP: 06440-160 - Barueri/SP Brasil. E-mail: kleber.silva1@hotmail.com

** Formação: Graduação em Administração pela Fundação Universidade Regional de Blumenau (FURB); Mestrado e Doutorado em Engenharia de Produção pela Universidade Federal de Santa Catarina (UFSC). Atividade profissional: Professora doutora e pesquisadora do Programa de Mestrado e Doutorado em Turismo e Hotelaria da Universidade do Vale do Itajaí (UNIVALI). Endereço profissional para correspondência: $5^{a}$ avenida, s./n., bloco 07, sala 206, Bairro dos Municípios. CEP: 88330-000 Balneário Camboriú/SC - Brasil. Telefone para contato: +55 47 3261-1315. Email: sara@univali.br
} 
on tourism in a more analytical way, highlighting the characteristics of the local reality in which these businesses are operating. It also presents an overview of the main contributions of studies on micro and small business in Brazil and internationally.

Key words: Tourism; Micro and small business; Bibliometric analysis; State of art. 


\section{INTRODUÇÃO}

As micro e pequenas empresas (MPE) têm constituído um assunto cada vez mais frequente nos debates em diferentes esferas da sociedade no Brasil. Esse protagonismo tem aumentado com a criação pelo Governo Federal da Secretaria da Micro e Pequena Empresa em 28 de março de 2013, o maior fortalecimento e desenvolvimento do Serviço Brasileiro de Apoio as Micro e Pequenas Empresas (SEBRAE) e o estabelecimento em 2011 da Associação Nacional de Estudos em Empreendedorismo e Gestão de Pequenas Empresas (ANEGEPE). (ANEGEPE, 2011; BRASIL, 2013; SEBRAE, 2012a).

As pequenas empresas são importantes agentes na transformação política e socioeconômica de uma nação (MORRISON; CARLSEN; WEBER, 2010). No setor do turismo, os últimos estudos publicados apontaram que as MPE representavam aproximadamente 97,2\% do total de empresas do setor no Brasil (IBGE, 2007), sendo que o segmento de meios de hospedagem estava formado em $90 \%$ por micro e pequenas empresas (EMBRATUR, 2006).

O aspecto positivo das MPE está na sua ramificação em diferentes localidades, principalmente em destinos mais afastados de grandes centros urbanos. Isso contribui na geração de empregos, especialmente em tempos de recessão, além de incentivar na inovação e no empreendedorismo, fomentando assim, o desenvolvimento econômico local e a competição de mercado (SMITH, 2006; TINSLEY; LYNCH, 2001).

Importantes revisões teóricas apontam que houve um avanço no número de publicações sobre MPE no turismo nas últimas duas décadas, porém com desenvolvimento mais lento (THOMAS; SHAW; PAGE, 2011), e operando na periferia em comparação com outras temáticas sobre turismo e hospitalidade (MORRISON; CONWAY, 2007).

Com base nessas afirmações, no presente trabalho se teve como objetivo principal analisar a produção científica sobre micro e pequenas empresas no turismo, no contexto brasileiro e internacional, entre 1990 e 2014. Busca-se apresentar a evolução do desenvolvimento das pesquisas, apontando as principais temáticas pesquisadas, definições de MPE adotadas, empresas objetos de estudo e localidades estudadas. 
Também será apresentado um panorama das principais contribuições dos estudos sobre o MPE em turismo no Brasil e no exterior.

A constituição dessa revisão bibliográfica contou com a utilização de duas importantes bases de dados e duas editoras de periódicos científicos internacionais, além das principais revistas científicas em turismo em circulação no Brasil e indexadas no portal "Publicações de Turismo". Os resultados possibilitaram conhecer o estado da arte do tema no mundo e no Brasil, servindo como referência tanto para pesquisadores que já estão inseridos na temática, quanto para aqueles que pretendem desenvolver pesquisas sobre MPE no turismo.

O artigo está estruturado em cinco partes: uma síntese teórica sobre as principais discussões a respeito da definição de micro e pequenas empresas; detalhamento da metodologia utilizada para o levantamento dos artigos brasileiros e estrangeiros; apresentação da evolução dos estudos internacionais e brasileiros sobre MPE no turismo; considerações finais com as principais conclusões e análises comparativas, além de apontamentos para futuras investigações.

\section{CONCEITO DE PEQUENA EMPRESA}

Verificou-se não existir uma definição internacional que seja aceita em comum acordo do que sejam micro e pequenas empresas (MORRISON; CARLSEN; WEBER, 2010; THOMAS; SHAW; PAGE, 2011; THOMAS, 2000). Constatou-se que os países adotam diferentes critérios, que podem variar conforme o número de funcionários (AUSTRALIA, 2011) ou a combinação entre receita anual e número de funcionários (EC, 2014; EGITO, 2009). Essas especificidades alteram-se de acordo com a finalidade de sua utilização, que pode originar-se de interesses específicos como legislação, financiamentos, estudos econômicos e demográficos.

No Brasil, há pelo menos três definições utilizadas para delimitar o que é uma MPE. A referência mais utilizada em assuntos legais é a Lei $123 / 06^{1}$ que trata do

\footnotetext{
${ }^{1}$ No ano de 2011 foi aprovada a lei complementar 139/2011, que institui a figura do "micro empreendedor individual" (MEI), que trata do pequeno empresário que trabalha por conta própria. Para ser um microempreendedor individual, é necessário faturar no máximo até $\mathrm{R} \$ 60.000,00$ por ano e não ter participação em outra empresa como sócio ou titular.
} 
"Estatuto Nacional da Microempresa e da Empresa de Pequeno Porte" (BRASIL, 2006), que utiliza a receita bruta anual para o enquadramento da empresa. O Banco Nacional do Desenvolvimento Econômico e Social (BNDES, 2012) utiliza-se de critério semelhante, porém com valores notavelmente superiores, cabendo à empresa enquadrarse para que sejam concedidas linhas de financiamento específicas de acordo com seu porte.

Dentre os critérios existentes, o que mais se aproxima do usualmente utilizado no exterior e em pesquisas acadêmicas é a definição do SEBRAE, que enquadra as empresas conforme o número de funcionários (SEBRAE, 2007). Esse valor altera-se conforme o segmento da empresa, a qual pode pertencer ao setor industrial e construção civil ou ao setor de serviços. A utilização deste critério é justificada pelo próprio órgão, como meio mais fácil para obtenção de dados e elaboração de estatísticas (SEBRAE, 2007).

As especificações de cada um dos critérios utilizados no Brasil estão no quadro 1, no qual se evidencia a diversidade de definições existentes no país. Essas diferenças vêm reforçar a função dos objetivos e interesses específicos de cada instituição e lei, que utilizam de classificação própria, sem conexão entre elas.

\begin{tabular}{|l|l|l|l|}
\hline QUADRO 1 - DEFINIÇÕES DE MICRO E PEQUENAS EMPRESAS NO BRASIL \\
\hline Lei 123/06 & $\begin{array}{l}\text { CRITÉRIO } \\
\text { Anual }\end{array}$ & MICROEMPRESA & PEQUENA EMPRESA \\
\hline BNDES & $\begin{array}{l}\text { Receita Bruta } \\
\text { Anual }\end{array}$ & $\begin{array}{l}\text { Igual ou inferior a } \\
\text { R\$ 360 mil }\end{array}$ & $\begin{array}{l}\text { R \$ } 360 \text { mil a } \\
\text { R } 3,6 \text { milhões }\end{array}$ \\
\hline $\begin{array}{l}\text { SEBRAE } \\
\text { Indústria e } \\
\text { construção }\end{array}$ & $\begin{array}{l}\text { Número de } \\
\text { funcionários }\end{array}$ & $\begin{array}{l}\text { Acima de } 2,4 \text { milhões } \\
\text { até } 16 \text { milhões }\end{array}$ \\
\hline $\begin{array}{l}\text { SEBRAE } \\
\text { Comércio } e \\
\text { serviços }\end{array}$ & $\begin{array}{l}\text { Número de } \\
\text { funcionários }\end{array}$ & Até 9 & 20 a 99 \\
\hline
\end{tabular}

Fonte: Adaptado de Brasil (2006), BNDES (2012) e SEBRAE (2012).

A variedade de definições tanto no Brasil quanto no exterior é vista por muitos autores como genéricas, uma vez que elas não evidenciam as especificidades dos diferentes setores econômicos. Outra dificuldade existente está na elaboração de estatísticas confiáveis que mensurem o impacto destas empresas na economia, 
prejudicando também na realização de estudos comparativos entre regiões e países (MORRISON; CONWAY, 2007; MORRISON, 1998; PAGE; FORER; LAWTON, 1999; THOMAS, 2000).

Alguns estudiosos (MORRISON; CARLSEN; WEBER, 2010; THOMAS; SHAW; PAGE, 2011; THOMAS, 2000) defendem que a simples adoção por pesquisadores de uma categorização oficial, não oferece uma contribuição significativa para o conhecimento. Eles defendem que junto a essa escolha deva ser feita uma fundamentação que justifique a utilização dessa classificação, assim como apresentar os contextos socioeconômicos da realidade investigada. Essa opinião entra em acordo com o importante debate da necessidade de analisar a complexidade que envolve as MPE no turismo, as quais precisam ser definidas de um modo mais analítico, que permita inferir sobre suas diferenças em relação às empresas maiores do mesmo setor ou de setores econômicos diferentes.

Nesse sentido, Ateljevic e Doorne (2004) e Elliot e Boshoff (2007), apontam a relação entre empreendedor e empresa como a principal diferença entre MPE e grandes empresas no turismo. Seus estudos analisam a influência dos valores pessoais dos proprietários na tomada de decisões e gestão do empreendimento, principalmente quando se trata de empresas familiares. Também merece destaque, Morrison e Conway (2007) tendo em vista que é um dos poucos estudos em que se propõe uma lista de características quantitativas e qualitativas consideradas por elas como essenciais para definir uma pequena empresa no turismo.

A heterogeneidade das empresas e de seus proprietários, além das características regionais apresentam desafios na busca por uma definição de MPE. A utilização de categorização pré-definida como quantidade de funcionários ou receita anual, é algo limitado, e que não expressa às especificidades das realidades investigadas. Por conta disso, considera-se a necessidade de reflexões epistemológicas sobre definições de MPE no turismo, de modo a oferecer subsídios para uma ampla análise do ambiente interno e externo, nos quais essas empresas estão inseridas. 


\section{METODOLOGIA}

Este estudo caracteriza-se como exploratório-descritivo, pois contou com levantamento e análise de um total de 196 artigos nacionais e internacionais, que serviram como base para a coleta de dados primários, que após serem tratados, possibilitaram a descrição do estado da arte sobre MPE no turismo. Utilizou-se de abordagens quantitativas e qualitativas, que contou com as estratégias de análise de estatística descritiva e análise de conteúdo de artigos científicos brasileiros e internacionais.

Os documentos foram selecionados por meio de consultas às bases de dados de periódicos científicos com acesso disponibilizado no portal de periódicos mantido pela Comissão de Aperfeiçoamento de Pessoal de Nível Superior (CAPES). Foram selecionadas duas bases de dados (ScienceDirect e EBSCO) e duas editoras (SAGE e Emerald) que indexam e publicam os principais periódicos de turismo na língua inglesa no mundo.

Para o levantamento dos artigos brasileiros, foram consultados os websites dos periódicos científicos em turismo até o mês de janeiro de $2015^{2}$, que se enquadrassem na avaliação B4, B3, B2, B1 no sistema Qualis na área de Administração, Ciências Contábeis e Turismo da CAPES, bem como os exemplares impressos das revistas, cujas edições estavam disponíveis na biblioteca da Universidade do Vale do Itajaí (UNIVALI) em Balneário Camboriú (Santa Catarina). Outras fontes como o website "Publicações de Turismo" (SANTOS, 2015), foram utilizados de modo a facilitar o processo de levantamento de artigos nos principais periódicos correntes e fora de circulação no Brasil.

O período definido para análise do estado da arte do tema foi de janeiro de 1990 a dezembro de 2014, período amplo, que compreende os primeiros artigos internacionais a tratarem de forma específica sobre o tema MPE no turismo, além de coincidir com o início da publicação da Revista Turismo em Análise, periódico mais antigo com publicação ininterrupta sobre turismo no Brasil.

\footnotetext{
2 Periódicos brasileiros consultados: Anais Brasileiros de Estudos Turísticos; Caderno Virtual de Turismo; Cultur - Revista de Cultura e Turismo; Intinerarium; Turismo e Sociedade; Revista Acadêmica Observatório de Inovação do Turismo; Revista Brasileira de Ecoturismo; Revista Brasileira de Pesquisa em Turismo; Revista Hospitalidade; Revista Iberoamericana de Turismo; Revista Rosa dos Ventos; Turismo - Visão e Ação; Turismo em Análise; Turismo: estudos e práticas.
} 
Durante o levantamento verificou-se haver um grande número de artigos, porém que não tinham como tema central MPE no turismo. Por conta disso, foram estabelecidos como critérios de seleção os artigos que tratassem de forma mais ampla e aprofundada o tema. Deste modo, foram feitas análises prévias do título, resumo e palavras-chave de cada artigo, caso houvesse alguma dúvida quanto ao conteúdo do trabalho, a introdução, a metodologia e as conclusões do mesmo eram lidas com maior profundidade, sendo descartados os artigos que tratassem do tema superficialmente. Também foram desconsideradas as notas de pesquisa, comentários de editores, relatos de eventos, estudos de caso e resenhas de livros.

Para o levantamento das publicações sobre MPE no turismo foram utilizadas as seguintes palavras-chave (em inglês e português) em buscas nos periódicos e nas bases de dados: micro; small; business; firms; enterprises; tourism; hospitality; hotel; micro; pequenas; empresas; negócios; turismo; hotelaria.

A coleta de dados resultou em um total de 175 e 21 artigos publicados, respectivamente, em periódicos internacionais e brasileiros. As seguintes informações de cada artigo foram tabuladas em uma planilha do software Microsoft Excel 2007: ano de publicação; tema central; definições de MPE adotadas nos trabalhos; locais investigados (países ou estados brasileiros); tipo do artigo (empírico ou teórico); tipos de empresas objetos de estudo. Esses dados foram identificados com base em informações explícitas no texto ou passíveis de serem inferidas pelos pesquisadores. Por fim, os artigos foram lidos parcialmente de modo a apresentar uma breve análise do estado da arte do tema. Os resultados são apresentados em forma de quadros, gráficos, figuras e tabelas.

Como limitação desta pesquisa, optou-se apenas por analisar artigos publicados em periódicos científicos. Não foi alvo deste estudo a publicação de livros, anais de eventos, nem dissertações e teses. Pelo fato das bases de dados internacionais conterem periódicos de diversas áreas do conhecimento, este levantamento não se restringiu apenas aos periódicos de turismo, mas também de outras áreas como administração, sustentabilidade ambiental, psicologia, entre outros, que eventualmente publicaram artigos sobre MPE no turismo. 


\section{PRODUÇÃO CIENTÍFICA INTERNACIONAL SOBRE MPE NO TURISMO}

Ao todo, entre 1990 a 2014, foram identificados 175 artigos publicados em periódicos internacionais na língua inglesa que tratavam de forma mais aprofundada do tema MPE no turismo. A evolução da literatura é um tanto quanto escassa ao longo da década de 1990, porém com acentuados avanços, seguidos de decréscimos na década de 2000 (gráfico 1).

GRÁFICO 1 - EVOLUÇÃO DA PUBLICAÇÃO DE ARTIGOS INTERNACIONAIS SOBRE MPE NO TURISMO (1990- 2014)

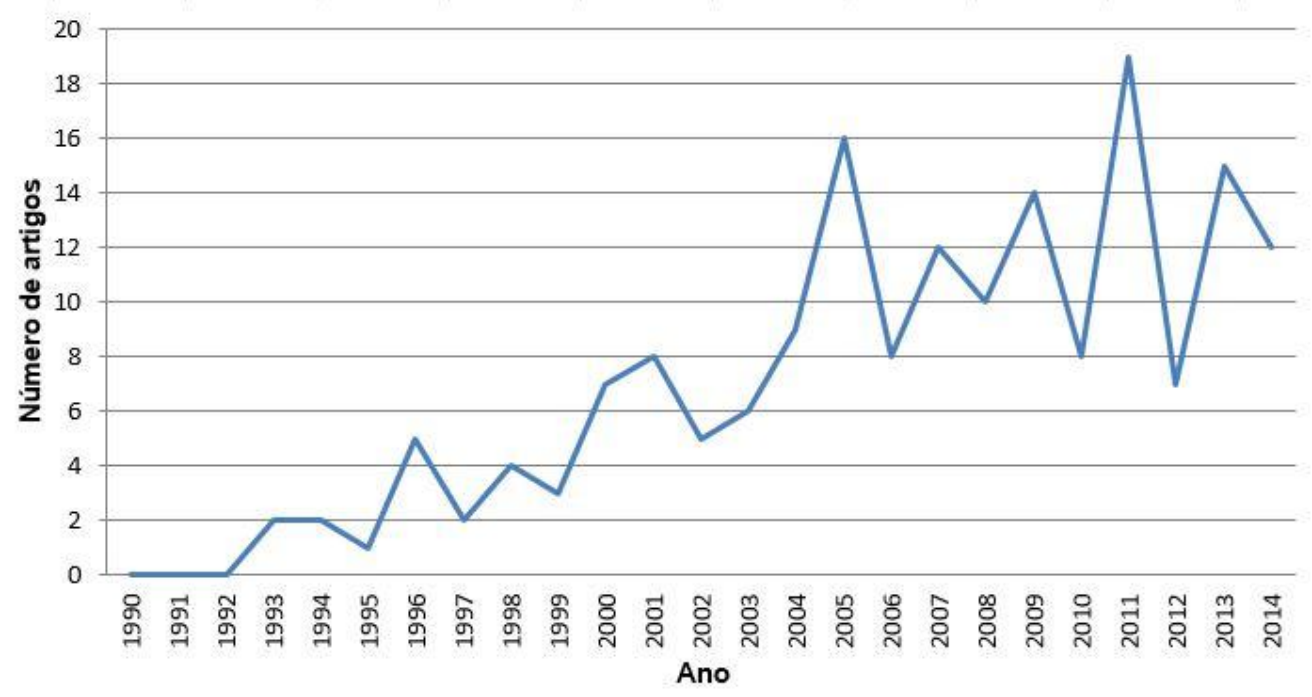

Fonte: Elaboração dos autores a partir das bases de dados e editoras consultadas: ScienceDirect, EBSCO, SAGE e Emerald.

Ao longo do período analisado ocorreu uma evolução irregular, com um grande aumento no número de publicações em determinados anos, seguidas de diminuições acentuadas nos anos posteriores. Por exemplo, em 2011 é o ano com maior crescimento totalizando 19 artigos, porém em 2012 o número de publicações cai para 7, e em 2013 volta a subir para 15, seguido por uma queda em 2014 para 12 artigos.

Esse total de artigos foi classificado conforme seu caráter empírico ou teórico, neste caso entende-se estudos empíricos como aqueles que incluem algum tipo de dado com análises estatísticas ou qualitativas, enquanto teóricos propõem-se a desenvolver ou elaborar uma análise geral de forma mais aprofundada visando traçar conclusões sobre o fenômeno estudado (VEAL, 2011). Do total de 175 artigos levantados, 157 são pesquisas empíricas, enquanto apenas 18 são teóricos. Na década de 1990 a publicação 
de artigos de caráter teórico foi esporádica e dispersa, enquanto que na década seguinte a incidência de artigos de caráter teórico foi mais contínua, apesar do pequeno número. Nos últimos três anos de análise da década de 2010 constata-se que nenhum artigo teórico foi publicado. Esse resultado está de acordo com as principais revisões teóricas, que apontam que o assunto sobre MPE no turismo ainda permanece subteorizado e com poucas pesquisas que propõem uma abordagem mais holística. A conclusão dessas revisões assinala que a literatura existente é rica em fatos e dados empíricos, porém pobre em teoria (GETZ; CARLSEN, 2005; MORRISON; CARLSEN; WEBER, 2010; THOMAS; SHAW; PAGE, 2011).

O principal motivo da falta de um corpo teórico mais aprofundado, segundo, segundo Thomas, Shaw e Page (2011) é a grande variedade de temas abordados, que representam iniciativas muitas vezes isoladas e com poucas pesquisas sobre o assunto. Apesar de que algumas áreas apresentarem maior desenvolvimento quanto às discussões, Morrison, Carlsen e Weber (2010) afirmam que ainda seria necessário um quadro teórico que integrasse esses trabalhos, que se apresentam em muitos casos dispersos, necessitando de uma maior consistência quanto às suas afirmações.

Em relação aos temas centrais dos artigos internacionais (tabela 1), verificou-se que algumas áreas apresentaram maior concentração de pesquisas, enquanto outras possuíam um menor corpo teórico ou em determinados casos, pesquisas com temáticas em estágio inicial de investigação no turismo.

TABELA 1 - TEMAS DOS ARTIGOS PUBLICADOS NO EXTERIOR

\begin{tabular}{lccllcc}
\hline Tema & $\begin{array}{c}\mathbf{N}^{\mathbf{0}} \mathbf{d e} \\
\text { Artigos }\end{array}$ & $\mathbf{\%}$ & Tema & $\begin{array}{c}\mathbf{N}^{\mathbf{0}} \mathbf{\text { de }} \\
\text { Artigos }\end{array}$ & $\%$ \\
\hline $\begin{array}{l}\text { Competitividade } \\
\text { Desempenho da empresa }\end{array}$ & 4 & 2,3 & Inovação & 6 & 3,4 \\
$\begin{array}{l}\text { Desenvolvimento } \\
\text { econômico da localidade }\end{array}$ & 6 & 3,4 & Marketing & 1 & 0,6 \\
Empreendedorismo & 22 & 12,6 & Políticas públicas & 41 & 23,4 \\
Ensaio teórico sobre MPE & 7 & 4,0 & Práticas de gestão & 1 & 0,6 \\
Estratégia do negócio & 3 & 1,7 & Recursos humanos & 20 & 11,4 \\
Estrutura organizacional & 2 & 1,1 & Redes de cooperação & 8 & 4,6 \\
Ética & 1 & 0,6 & Relações públicas & 1 & 0,6 \\
Financiamento & 2 & 1,1 & Responsabilidade & 3 & 1,7 \\
Gestão do conhecimento & 2 & 1,1 & $\begin{array}{l}\text { Sustentabilidade } \\
\text { ambiental }\end{array}$ & 15 & 8,6 \\
\hline
\end{tabular}




\begin{tabular}{|c|c|c|c|c|c|}
\hline Tema & $\begin{array}{c}\mathbf{N}^{\mathbf{0}} \text { de } \\
\text { Artigos }\end{array}$ & $\%$ & Tema & $\begin{array}{c}\mathbf{N}^{\mathbf{0}} \text { de } \\
\text { Artigos }\end{array}$ & $\%$ \\
\hline $\begin{array}{l}\text { Influências internas e } \\
\text { externas na empresa }\end{array}$ & 10 & 5,7 & & & \\
\hline \multicolumn{6}{|c|}{ TOTAL DE ARTIGOS } \\
\hline \multicolumn{6}{|c|}{$175(100 \%)$} \\
\hline
\end{tabular}

Fonte: Elaboração dos autores a partir das bases de dados e editoras consultadas: ScienceDirect, EBSCO, SAGE e Emerald.

Dentre as áreas mais estabelecidas, identificou-se o marketing com uma participação de 23,4\% no total de pesquisas sobre MPE no turismo. Em seguida destacaram-se pesquisas sobre empreendedorismo com 12,6\%, recursos humanos com $11,4 \%$ e desempenho de pequenas empresas e sustentabilidade ambiental, ambos com 8,6\%. Esses temas podem ser classificados como áreas de estudo estabelecidas no turismo, pois se mostram mais produtivas, com maior envolvimento de pesquisadores, apesar de precisarem de maior incremento teórico (THOMAS; SHAW; PAGE, 2011).

Por outro lado, influências internas e externas na empresa $(5,7 \%)$, redes de cooperação (4,6\%), ensaios teóricos sobre MPE (4\%), desenvolvimento econômico da localidade $(3,4 \%)$, inovação $(3,4 \%)$, políticas públicas $(2,9 \%)$ e competitividade $(2,3 \%)$ representaram temas emergentes. Essas são pesquisas limitadas, mas com dados empíricos que podem fomentar o desenvolvimento de novos estudos, que venham a incrementar essas análises. Os demais temas na tabela receberam um pequeno foco de atenção. Apesar de representarem estudos isolados, eles sugerem a perspectiva do surgimento de áreas de pesquisas em estágio inicial.

Apesar de a literatura ser apresentada como dispersa e com pouco diálogo entre si (GETZ; CARLSEN, 2005; MORRISON; CARLSEN; WEBER, 2010; THOMAS; SHAW; PAGE, 2011), notou-se a existência de algumas relações envolvendo a variedade de trabalhos levantados. Isto possibilita criar um importante quadro de semelhanças e diferenças, que em conjunto permitam um melhor conhecimento do estado da produção científica desenvolvida na área.

Uma grande parcela de estudos que analisaram as características dos empreendedores de MPE no turismo destacaram principalmente questões envolvendo suas motivações. Muitas MPE no setor do turismo são, em diferentes níveis, afetadas fortemente por razões pessoais e de estilo de vida de seus proprietários. Esse fato tem destaque nas discussões que apresentam os proprietários que priorizam os objetivos de 
estilo de vida, em detrimento das necessidades de ganhos econômicos (MORRISON; TEIXEIRA, 2004). Outra área de estudos no empreendedorismo que tem chamado a atenção dos pesquisadores são as empresas familiares, principalmente devido às suas complexas dimensões humanísticas que afetam MPE (GETZ; CARLSEN, 2005).

O tema de recursos humanos enfoca em sua maioria a percepção de gestores quanto a qualificação de seus funcionários, além de mostrar práticas usuais nas relações no trabalho. Ganha destaque a percepção dos funcionários (PETERS, 2005) e dos empreendedores (PITTAWAY, 2005) quanto a satisfação e necessidades no trabalho.

Pesquisas sobre sustentabilidade ambiental, apesar de ser um tema bastante abordado na literatura geral de turismo, recebe pouca atenção em relação às MPE. Os maiores destaques foram os estudos que enfocaram as percepções e barreiras quanto a adoção de práticas ambientalmente sustentáveis nos negócios (KASIM, 2009; RADWAN, JONES, MINOLI, 2010; VERNON et al., 2003).

Os trabalhos de marketing em grande parte enfocaram o impacto da utilização de tecnologias da comunicação e informação (TICs) em pequenas empresas. Destaca-se o uso da internet e os fatores que influenciam sua adoção e a consequente contribuição para o crescimento e sucesso da empresa (EL-GOHARY, 2012; IRVINE, ANDERSON, 2008).

Os artigos que analisaram as influências que afetam o desenvolvimento de micro e pequenas empresas de turismo ressaltaram a existência de uma diversidade de fatores que variam desde ineficiências operacionais internas (SHARMA; UPNEJA, 2005), até questões externas relacionadas ao ambiente de mercado e apoio governamental (WAN; KONG, 2008). Apesar da importância do tema gestão empresarial, constatou-se que poucas pesquisas buscaram analisar as características de gerenciamento interno de MPE, dentre os assuntos tratados, os que mais se destacaram são os trabalhos que investigaram as fontes de financiamentos utilizadas por MPE no turismo (ATELJEVIC; DOORNE, 2004; OZER; YAMAK, 2000).

Foi fortemente apontada na literatura sobre MPE no turismo, a importância da formação de redes e alianças estratégicas entre pequenas empresas para aumentar a competitividade do setor. Também foi defendido o incentivo a cooperação entre empresas visando o auxílio na solução de problemas em conjunto (HWANG; LOCKWOOD, 2006). 
Os autores das pesquisas existentes adotaram uma variedade de definições para referirem-se a MPE no turismo. Por meio da leitura parcial de cada artigo, permitiu-se que fosse constatado que do total de 175 artigos levantados, em torno de $49 \%$ deles não apresentaram os critérios escolhidos ou base teórica para a definição de uma MPE no Turismo. Em relação aos autores que utilizaram alguma definição, cerca de $33 \%$ eram critérios dos próprios autores do artigo, o que representava uma variedade de critérios, muitas vezes baseados em escolhas pessoais ou citação de outros autores. Em menor escala com $18 \%$ estavam os trabalhos, que adotaram definições oficiais de acordo com o país de origem de cada pesquisa, era comum a presença de critérios da Comissão Europeia, "UK Trade and Industry" do Reino Unido e o "Australia Bureau of Statistics" da Austrália.

Essa disparidade na adoção de uma definição de MPE no turismo revela que a academia utiliza-se de forma deliberada o termo "pequena empresa" para referir-se a uma variedade de realidades (THOMAS, 2000). Na maioria dos casos não são apresentados os critérios utilizados, e as devidas justificativas de sua adoção, havendo em certos estudos total divergência quanto às conceituações utilizadas.

Essa falta de conexão entre as definições adotadas vem evidenciar claramente as dificuldades de se fazer análises comparativas entre os trabalhos. Além da falta de consistência em oferecer subsídios para uma discussão mais teórica com base nas definições utilizadas. Essa realidade sugere a necessidade de discutir o tema em bases epistemológicas.

O levantamento dos destinos objeto de estudo dos artigos, permitiu que fosse feita a figura 1, que apresenta uma distribuição geográfica com o destaque para os 44 diferentes países com pesquisas sobre MPE no turismo. 
FIGURA 1- PAÍSES COM PESQUISAS SOBRE MPE EM TURISMO ( $\mathrm{N}^{\circ}$ DE ARTIGOS POR PAÍS E PARTICIPAÇÃO \% DO CONTINENTE)

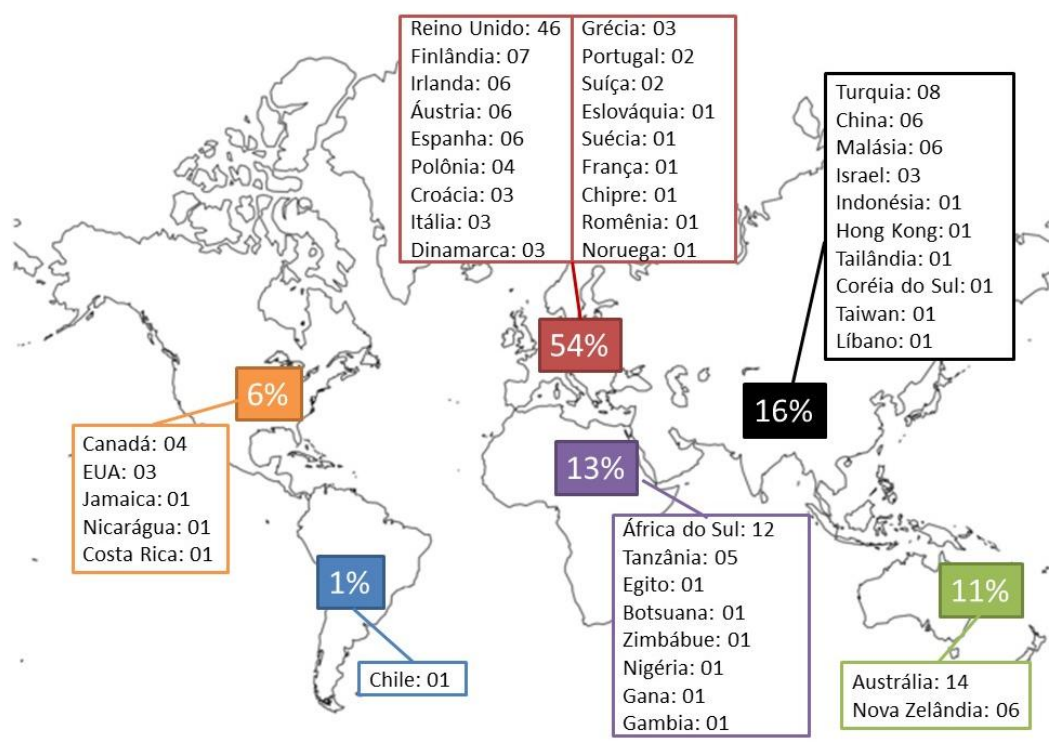

Fonte: Elaboração dos autores a partir das bases de dados e editoras consultadas: ScienceDirect, EBSCO, SAGE e Emerald ${ }^{3}$.

Verificou-se haver participação de todos os continentes, no entanto percebeu-se uma forte variação quanto ao número de estudos analisando a realidade de cada país.

A maioria dos estudos foi proveniente da Europa (54\%), com grande destaque para o Reino Unido que continha o maior número de artigos publicados, 46 do total. Este país pode ser considerado como referência em pesquisas sobre este tema, pois conta com estudos que analisam diferentes aspectos de MPE em turismo. Estas pesquisas servem como interessante base para a discussão e comparação com resultados de outros países, além do desenvolvimento de novos estudos. Friel (1999) explica que a forte presença do Reino Unido, principalmente em pesquisas publicadas na década de 90 foi devido ao amplo apoio governamental no desenvolvimento de pesquisas sobre MPE, além da implantação de um centro específico de pesquisas sobre este tema na Leeds Metropolitan University, que não está em atividade atualmente.

A Ásia concentrou $16 \%$ do total de artigos, seguida pela África (13\%) e Oceania (11\%). Esses resultados revelam a predominância de estudos provenientes de países desenvolvidos. A literatura sobre MPE em turismo em países em desenvolvimento ainda é relativamente pequena (ROGERSON, 2005), sendo a maior contribuição originária da África do Sul (12), Turquia (8), China (6) e Malásia (6). Porém alguns

\footnotetext{
3 Fonte da figura de fundo: <http://www.lacasainfantil.com/materiales-y-recursos/mapa-mundi-enblanco>.
} 
autores vislumbram para o futuro um aumento no interesse da comunidade científica em empreender pesquisas sobre projetos de organizações mundiais, que têm fomentado o desenvolvimento de MPE em países emergentes (ROGERSON, 2008; THOMAS; SHAW; PAGE, 2011). É apontado como marcante em pesquisas em países em desenvolvimento, a diversidade de micro e pequenas empresas inseridas nas mais diferentes realidades, que servem como importante incremento na discussão já conhecida em países desenvolvidos (THOMAS; SHAW; PAGE, 2011).

Com relação ao tipo de empresa que foi objeto de estudo dos trabalhos (tabela 2), 156 artigos tiveram como foco a análise de um ou mais segmentos de empresas, enquanto 19 pesquisas eram ensaios teóricos sem referência a empresas específicas. Os resultados apontam que 59,6\% dos autores dos artigos analisaram em um mesmo conjunto diferentes setores do turismo, como por exemplo, hotéis, restaurantes, meios de transporte, agências de viagens e operadores de turismo. Geralmente estes estudos não apresentaram as diferenças e características particulares de cada setor estudado. Isso influencia com que conclusões generalizadas sejam feitas, normalmente baseadas em análises de resultados que acabam por mascarar as particularidades de cada setor.

TABELA 2 - TIPOS DE EMPRESAS OBJETOS DE ESTUDO

\begin{tabular}{lcc}
\hline \multicolumn{1}{c}{ Segmento da empresa } & $\begin{array}{c}\mathbf{N}^{\mathbf{0}} \mathbf{\text { de }} \\
\mathbf{a r t i g o s}\end{array}$ & $\mathbf{\%}$ \\
\hline Vários segmentos & 93 & 59,6 \\
Meios de hospedagem & 54 & 34,6 \\
Agências de viagens & 4 & 2,6 \\
Restaurantes & 3 & 1,9 \\
Operadores de turismo & 1 & 0,6 \\
Agências de locação de imóveis & 1 & 0,6 \\
TOTAL & $\mathbf{1 5 6}$ & $\mathbf{1 0 0}$ \\
\hline
\end{tabular}

Fonte: Elaboração dos autores a partir das bases de dados e editoras consultadas: ScienceDirect, EBSCO, SAGE e Emerald.

Dos setores analisados de forma isolada, os meios de hospedagem receberam maior atenção dos pesquisadores. Aproximadamente 34,6\% dos artigos, oferecem análises sobre este setor. Com menor participação estão áreas como agências de viagens, restaurantes e operadores de turismo. Isso revela a necessidade de mais estudos específicos abordando esses setores separadamente, de modo a oferecer dados que possibilitem melhor entendimento das especificidades das empresas que compõem estas 
áreas. Assim como setores com foco em segmentos específicos (SCHOTT, 2007), como é o caso de empresas de turismo de aventura.

As diferenças de desempenho em três atividades no turismo (meios de hospedagem, atrações turísticas, atividades de recreação) em Israel foram analisadas por Reichel e Haber (2005), que demonstraram que cada um possui características e condições inerentes as suas áreas de atuação. Por conta disso, os autores defenderam que as especificidades de cada atividade no turismo, sejam tratadas de forma mais aprofundada e separada das demais, permitindo assim, acompanhar o desempenho de cada segmento. Isso facilita com que comparações mais assertivas sejam feitas entre eles.

\section{PRODUÇÃO CIENTÍFICA BRASILEIRA SOBRE MPE NO TURISMO}

Foram identificados 21 artigos sobre micro e pequenas empresas no turismo, publicados em periódicos brasileiros no período de 1990 a 2014. No entanto, o levantamento, conforme consta no gráfico 2, revela que a pesquisa sobre esse tema no Brasil é recente com o primeiro estudo publicado no ano de 2004. Desde então, houve um aumento pequeno no número de publicações, seguido de um período sem publicação nos anos de 2007 e 2008. Os anos posteriores apresentam uma ascensão no número de artigos, com o ano de 2012 como a maior concentração com um total de 4 artigos, seguida por uma queda acentuada no ano de 2013 e pequeno crescimento em 2014.

GRÁFICO 2 - EVOLUÇÃO DA PUBLICAÇÃO DE ARTIGOS BRASILEIROS SOBRE MPE NO TURISMO (2004- 2014)

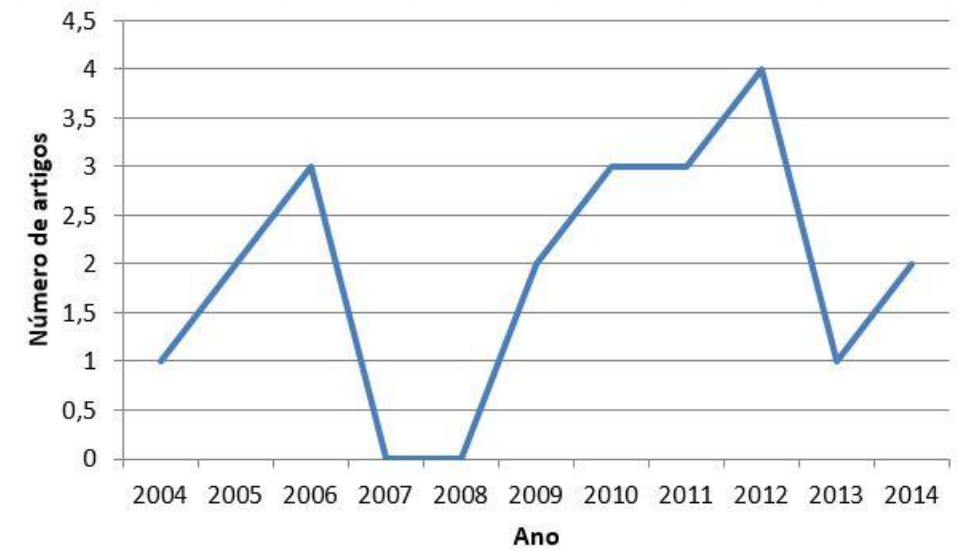

Fonte: Elaboração dos autores a partir da consulta aos periódicos brasileiros e a base de dados Publicações de Turismo. 
A classificação dos artigos conforme seu caráter empírico ou teórico, publicados durante o período estabelecido evidencia que a realidade da pesquisa no Brasil foi semelhante à internacional, ou seja, há grande presença de estudos empíricos em detrimento de teóricos. No caso brasileiro, percebeu-se que o desenvolvimento de uma discussão teórica só chamou atenção da comunidade científica no ano de 2012.

Em relação às temáticas das pesquisas publicadas no Brasil (tabela 3), viu-se que não houve uma grande variedade de assuntos, como é verificado na literatura internacional. Os trabalhos brasileiros foram focados em temas centrais, envolvendo aspectos de formação de redes e cooperação entre empresas, motivação e características dos empreendedores.

TABELA 3 - TEMAS DOS ARTIGOS PUBLICADOS NO BRASIL

\begin{tabular}{lclc}
\hline Tema & $\begin{array}{c}\mathbf{N}^{\mathbf{0}} \text { de } \\
\text { Artigos }\end{array}$ & Tema & $\begin{array}{c}\mathbf{N}^{\mathbf{0}} \mathbf{d e} \\
\text { Artigos }\end{array}$ \\
\hline Comunicação organizacional & 1 & Qualidade dos serviços & 1 \\
Desempenho da empresa & 2 & Recursos humanos & 1 \\
Empreendedorismo & 5 & $\begin{array}{l}\text { Redes e cooperação } \\
\text { Tecnologias da } \\
\text { comunicação e } \\
\text { informação }\end{array}$ & 8 \\
Estratégia do negócio & 1 & 1 \\
Marketing & 1 &
\end{tabular}

\section{TOTAL DE ARTIGOS}

21

Fonte: Elaboração dos autores a partir da consulta aos periódicos brasileiros e a base de dados Publicações de Turismo.

Diferente da literatura internacional, a maior parte das pesquisas desenvolvidas no Brasil foi concentrada nos temas redes e cooperação (8 artigos) e empreendedorismo (5 artigos) em MPE de turismo. Em participação menor, está desempenho da empresa com 2 artigos do total, pontua-se que a temática "marketing" (1) e em conjunto com "recursos humanos" (1) foram os que apresentaram o maior número de publicações de artigos internacionais.

Dentre os assuntos pesquisados verificou-se haver um considerável número de pesquisas abordando as atitudes e motivações dos empreendedores, e também as competências necessárias para o desenvolvimento de suas funções no negócio. Também destacam-se os estudos, que analisaram a realidade da formação de redes de MPE em alguns destinos brasileiros. 
As pesquisas sobre motivações dos empreendedores foram predominantemente realizadas em países desenvolvidos, revelando a forte presença de empreendedores que iniciaram seus negócios motivados pela busca de um estilo de vida. Nessa perspectiva, o trabalho de Costa e Nascimento (2010) merece destaque por demonstrar uma realidade diferente presente no Brasil. Os autores desvendaram que as principais motivações de empresários estabelecidos nas cidades de Jericoacoara (CE), Parnaíba (PI) e Barreirinhas (MA) foram de caráter econômico, sendo que os entrevistados ressaltaram ver no turismo um negócio lucrativo nas localidades.

Verificaram-se as definições de MPE utilizadas pelos 21 estudos publicados em periódicos de turismo no Brasil. Assim como na literatura internacional, constatou-se a existência de um grande número de artigos que não apresentaram uma definição de MPE (9 artigos). Em seguida, percebeu-se a utilização do critério de número de funcionários proposto pelo SEBRAE (10 artigos). Em menor participação (2 artigos) ocorreu a utilização de critérios estabelecidos pelos próprios autores dos trabalhos.

Quanto aos contextos geográficos dos estudos, teve-se 11 estados com pesquisas sobre MPE no turismo. O estado do Paraná se destacou com o maior número de artigos (5), que analisaram a realidade da cidade de Curitiba, em seguida encontra-se o estado de Sergipe com 3 artigos. A presença desses estados pode ser justificada, principalmente, devido à autoria dos artigos, cuja pesquisadora Rivanda de Meira Teixeira esteve vinculada à Universidade Federal do Paraná, tendo posteriormente mudado para a Universidade Federal de Sergipe ${ }^{4}$.

Os demais estados possuem entre 1 ou 2 estudos, figurando, além do Distrito Federal (1), os seguintes estados: Alagoas (1), Ceará (2), Maranhão (2), Mato Grosso do Sul (1), Minas Gerais (1), Piauí (2), Rio de Janeiro (1), São Paulo (2).

Quando analisadas as regiões, houve uma clara concentração de estudos descrevendo a realidade do Nordeste, seguida pelo Sul. A região Norte foi a única que não contou com nenhum artigo.

No levantamento também se encontrou um artigo analisando a realidade de países europeus, e como comentado anteriormente, um estudo bibliométrico (XAVIER et al., 2012), que não analisou nenhum destino turístico.

\footnotetext{
${ }^{4}$ Informação consultada no Currículo Lattes da autora: <http://lattes.cnpq.br/9843881511690849>.
} 
A grande maioria das pesquisas sobre MPE no turismo (tabela 4) tiveram os meios de hospedagem como principal objeto de estudo, com aproximadamente 13 artigos. Em seguida houve preferência por 6 trabalhos em analisar diferentes segmentos de empresas de um destino turístico. Essas empresas compreendiam meios de hospedagem, restaurantes, agências de receptivo e de transportes turísticos. Por fim, houve a presença de um trabalho que analisou as empresas de turismo de aventura.

TABELA 4 - SETORES OBJETOS DE ESTUDO

\begin{tabular}{ll}
\hline Segmento da empresa & $\mathbf{N}^{\mathbf{0}}$ de artigos \\
\hline Meios de hospedagem & 13 \\
Vários segmentos & 6 \\
Empresas de aventura & 1 \\
TOTAL & $\mathbf{2 0}$ \\
\hline Fonte: Elaboração dos autores a partir da consulta aos \\
periódicos brasileiros e a base de dados Publicações de \\
Turismo.
\end{tabular}

Esses resultados demonstraram a preferência da academia brasileira em desenvolver pesquisas sobre meios de hospedagem

\section{CONSIDERAÇÕES FINAIS}

Nessa pesquisa se teve como objetivo apresentar um panorama das pesquisas sobre micro e pequenas empresas em turismo publicadas nos principais periódicos científicos brasileiros e internacionais.

A análise do estado da arte em conjunto com importantes revisões internacionais permite afirmar que a literatura sobre MPE no turismo precisa de maior embasamento teórico. Principalmente quanto a definição e caracterização das MPE no setor do turismo, dado que a maioria dos artigos internacionais e brasileiros pesquisados não adotaram uma definição em seus estudos, ou quando adotaram os critérios divergem entre si. Por conta disso, as pesquisas futuras necessitam analisar as MPE de um modo mais analítico, ressaltando as características da realidade na qual essas empresas estão inseridas.

A maioria dos trabalhos existentes é proveniente de países desenvolvidos, com destaque para o Reino Unido e Austrália, isso faz com que haja necessidade de 
ponderação quanto a utilização destes trabalhos como referência, dada a existência de realidades muitas vezes distintas em comparação com países em desenvolvimento. Porém, notou-se que países emergentes, como África do Sul e a Turquia, têm ganhado destaque com pesquisas sobre este tema.

No que se refere aos tipos de empresas objetos de estudos, notou-se uma clara preferência da academia em analisar em um mesmo conjunto diferentes setores empresariais. Isso vem em contraponto aos apontamentos de estudos (REICHEL; HABER, 2005; THOMAS, 2000), que defendem que cada setor no turismo deva ser tratado de forma separada, permitindo assim, uma análise mais aprofundada das especificidades de cada segmento. Neste sentido, verificou-se que o setor de meios de hospedagem é o que contou com o maior número de pesquisas tanto na literatura brasileira, quanto internacional sobre MPE no turismo.

No Brasil as pesquisas sobre MPE no turismo são recentes, o que oferece oportunidades para o desenvolvimento de pesquisas que venham a contribuir para o melhor entendimento do tema. Notou-se a preferência dos pesquisadores brasileiros nos temas redes e cooperação e empreendedorismo, além de maior concentração de estudos analisando a realidade das regiões Nordeste e Sul. Além disso, não foram encontrados trabalhos retratando a realidade brasileira nos principais periódicos em língua inglesa.

Sugere-se que esta pesquisa seja ampliada no futuro, e que uma análise mais abrangente em periódicos brasileiros ligados à área de administração e serviços sejam utilizados como referência, também recomenda-se que novos levantamentos sejam feitos, abrangendo também periódicos publicados no idioma espanhol e outros idiomas.

Dessa forma, permanece a necessidade de maior atenção no aprofundamento de estudos sobre micro e pequenas empresas no turismo, reafirmando que futuras pesquisas devam estar em constante diálogo com trabalhos desenvolvidos no exterior, de modo a identificar e analisar diferentes realidades e situações semelhantes.

O desenvolvimento de uma base teórica e empírica mais consolidada será de fundamental importância para o desenvolvimento de políticas públicas e programas de apoio às micro e pequenas empresas no turismo no Brasil. 


\section{REFERÊNCIAS}

ANEGEPE. Estatuto de constituição da Associação Nacional de Estudos em Empreendedorismo e Gestao de Pequenas Empresas - ANEGEPE. Disponível em: <http://www.anegepe.org.br/download/final-estatuto-anegepe-22-02-201111.pdf>. Acesso em: 20 nov. 2014.

ATELJEVIC, J.; DOORNE, S. Diseconomies of scale: A study of development constraints in small tourism firms in central New Zealand. Tourism and Hospitality Research, v. 5, n. 1, p. 5-24, 2004.

AUSTRALIA. Key statistics: australian small business. 2011. ed. Canberra: Commonwealth of Australia, 2011. p. 1-56

BNDES. Apoio às micro, pequenas e médias empresas. Rio de Janeiro: BNDES, 2012. p. $1-29$

BRASIL. Lei Complementar $\mathrm{N}^{0} 123$, de 14 de dezembro de 2006: Estatuto Nacional da Microempresa e da Empresa de Pequeno Porte. . 2006.

BRASIL. Lei $N^{0}$ 12.792, de 28 de março de 2013: criação da Secretaria da Micro e Pequena Empresa. . 2013.

COSTA, H. A.; NASCIMENTO, E. P. DO. Motivações para empreender no turismo: um estudo sobre micro e pequenas empresas na Costa Norte (CE, MA, PI). Turismo Visão e Ação, v. 12, n. 3, p. 314-330, 2010.

EC. What is an SME? (online document). Disponível em: <http://ec.europa.eu/enterprise/policies/sme/files/sme_definition/sme_user_guide_en.pd f>. Acesso em: 31 maio. 2014.

EGITO. Law number 141 dor the year 2009, the small business enterprises development law. . 2009.

EL-GOHARY, H. Factors affecting E-Marketing adoption and implementation in tourism firms: an empirical investigation of egyptian small tourism organizations. Tourism Management, v. 33, n. 5, p. 1256-1269, 2012.

ELLIOTT, R.; BOSHOFF, C. The influence of the owner-manager of small tourism businesses on the success of internet marketing. South Africa Journal of Business Management, v. 38, n. 3, p. 15-27, 2007.

EMBRATUR. Meios de hospedagem: estrutura de consumo e impactos na economia. São Paulo: Ministério do Turismo, 2006. p. 1-101

FRIEL, M. Marketing practice in small tourism and hospitality firms. International Journal of Tourism Research, v. 1, p. 97-109, 1999. 
GETZ, D.; CARLSEN, J. Family business in tourism: state of art. Annals of Tourism Research, v. 32, p. 237-258, 2005.

HWANG, L.-J. J.; LOCKWOOD, A. Understanding the challenges of implementing best practices in hospitality and tourism SMEs. Benchmarking: An International Journal, v. 13, n. 3, p. 337-354, 2006.

IBGE. Economia do Turismo: análise das atividades características do turismo 2007. Rio de Janeiro: IBGE, 2007.

IRVINE, W.; ANDERSON, A. R. ICT (information communication technology), peripherality and smaller hospitality businesses in Scotland. International Journal of Entrepreneurial Behaviour \& Research, v. 14, n. 4, p. 200-218, 2008.

KASIM, A. Managerial attitudes towards environmental management among small and medium hotels in Kuala Lumpur. Journal of Sustainable Tourism, v. 17, n. 6, p. 709$725,2009$.

MORRISON, A. Small firm statistics: a hotel sector focus. The Service Industries Journal, v. 18, n. 1, p. 132-142, 1998.

MORRISON, A.; CARLSEN, J.; WEBER, P. Small tourism business research: change and evolution. International Journal of Tourism Research, v. 12, p. 739-749, 2010.

MORRISON, A.; CONWAY, F. The status of the small hotel firm. The Service Industries Journal, v. 27, n. 1, p. 47-58, 2007.

MORRISON, A.; TEIXEIRA, R. M. Small business performance: a tourism sector focus. Journal of Small Business and Enterprise Development, v. 11, n. 2, p. 166$173,2004$.

OZER, B.; YAMAK, S. Self-sustaining pattern of finance in small businesses $\square$ : evidence from Turkey. International Journal of Hospitality Management, v. 19, p. 261-273, 2000.

PAGE, S. J.; FORER, P.; LAWTON, G. R. Small business development and tourism: terra incognita? Tourism Management, v. 20, n. 4, p. 435-459, 1999.

PETERS, M. Entrepreneurial skills in leadership and human resource management evaluated by apprentices in small tourism businesses. Education + Training, v. 47, n. 8, p. 575-591, 2005.

PITTAWAY, L. "Mind the gap": graduate recruitment in small businesses. International Small Business Journal, v. 23, n. 4, p. 403-426, 2005.

RADWAN, H. R. I.; JONES, E.; MINOLI, D. Managing solid waste in small hotels. Journal of Sustainable Tourism, v. 18, n. 2, p. 175-190, 2010. 
REICHEL, A.; HABER, S. A three-sector comparison of the business performance of small tourism enterprises: an exploratory study. Tourism Management, v. 26, n. 5, p. 681-690, 2005.

ROGERSON, C. M. Unpacking tourism SMMEs in South Africa: structure, support needs and policy response. Development Southern Africa, v. 22, n. 5, p. 623-642, 2005.

ROGERSON, C. M. Shared growth in urban uourism: evidence from Soweto, South Africa. Urban Forum, v. 19, n. 4, p. 395-411, 2008.

SCHOTT, C. Selling adventure tourism $\square$ : a distribution channels perspective. International Journal of Tourism Research, v. 9, p. 257-274, 2007.

SEBRAE. Fatores condicionantes e taxas de sobrevivência e mortalidade das micro e pequenas empresas no brasil 2003-2005. Brasília: SEBRAE, 2007. p. 1-60

SEBRAE. Sistema SEBRAE - direcionamento estratégico 2013-2022. Disponível em: <http://www.sebrae.com.br/Sebrae/Portal Sebrae/Anexos/Direcionamento Estrategico 2022.pdf>. Acesso em: 20 nov. 2014a.

SEBRAE. Anuário das pesquisas sobre as micro e pequenas empresas (2011)Brasília, 2012b.

SHARMA, A.; UPNEJA, A. Factors influencing financial performance of small hotels in Tanzania. International Journal of Contemporary Hospitality Management, v. 17, n. 6, p. 504-515, 2005.

SMITH, S. L. J. How big, how many? Enteprise size distributions in tourism and other industries. Journal of Travel Research, v. 45, n. 1, p. 53-58, 2006.

THOMAS, R. Small firms in the tourism industry: some conceptual issues. International Journal of Tourism Research, v. 2, p. 345-353, 2000.

THOMAS, R.; SHAW, G.; PAGE, S. J. Understanding small firms in tourism: a perspective on research trends and challenges. Tourism Management, v. 32, p. 963976, 2011.

TINSLEY, R.; LYNCH, P. Small tourism business networks and destination development. International Journal of Hospitality Management, v. 20, n. 4, p. 367$378,2001$.

VEAL, A. J. Metodologia de pesquisa em lazer e turismo. 1. ed. São Paulo: Aleph, 2011.

VERNON, J. et al. The "greening" of tourism micro-businesses: outcomes of focus group investigations in South East Cornwall. Business Strategy and the Environment, v. 12, n. 1, p. 49-69, jan. 2003. 
WAN, P. Y. K.; KONG, W. H. An assessment of the business environment for small Tourism ventures development in the Gaming City of Macao. Journal of China Tourism Research, v. 4, n. 3, p. 297-318, 2008.

XAVIER, T. R. et al. A relação entre redes e turismo: uma análise bibliométrica sobre a emergência de um novo paradigma no planejamento turístico. Turismo \& Sociedade, v. 5, n. 2, p. 443-465, 2012.

Recebido em: 15-04-2014.

Aprovado em: 15-05-2014. 